\title{
EXPERIMENTAL AND NUMERICAL ANALYSIS OF ECCENTRICALLY LOADED BEAM-COLUMNS
}

The beam-column subjected to the combination of the axial force and the bending moments is an example of the very complicated task from the viewpoint of the design resistance verification. The general theoretical solution of the resistance assessment of that beam-column is not suitable for practical use due to complicated and non-general form. Then, the standard simplified approach is used. Therefore, the paper deals with the experimental and numerical analyses of the resistances of eccentrically loaded beam-columns and their comparison with the standard approaches. Discussion of results and conclusions related to further research direction are also presented in this paper.

Keywords: Eccentrically loaded beam-column, beam-column resistance, experimental analysis, numerical study.

\section{Introduction}

The verification of the beam-column resistance subjected to the axial force in combination with the bending moments is a very complex task from the viewpoint of design practice. Therefore, the standard approach [1] to beam-column resistance assessment is based on the simplified model of substitute member whose accuracy and suitability is controversial in the special cases of member load effects and member boundary conditions. Then, the determination of the beam-column resistance and its verification need necessarily to be more precise and complex, supported by the numerical calculations or experimental analyses. From this point of view, the paper presents results of experimental and numerical analyses of the determination of the beam-column resistance and its verification compared to the standard approaches [1].

\section{Theoretical background}

In general, the bending and torsional moments' equilibrium of the pin-ended beam-column with the double symmetric constant cross-section, initially imperfect about both axes subjected to the constant compressive axial force and bending moments, could be described by means of the system of differential equations presented, e.g., in [2] and [3]. Generally, the system of differential equations represents system with non-constant coefficients whose solution cannot be found in an analytical form. In the case when only the axial force is acting eccentrically with respect to both cross-sectional axes and the eccentricities are constant within the length of the beam-column, the bending moments $M_{y}$ and $M_{z}$ have constant shapes. Then, the exact solution in the analytical form should only be found. The system of equations becomes more inhomogeneous when the initial equivalent geometric imperfections in the form of initial bows about axes y-y and z-z, and initial cross-sectional rotation are implemented into the system. Thus, buckling resistance of the initially imperfect beamcolumn with double symmetric constant cross-section, subjected to eccentrically axial compressive force $N$, causing bi-axial bending moments $M_{y}$ and $M_{z}$ in the two principal cross-section planes of loading, can be described by the system of differential equations in the following forms

$$
\begin{aligned}
& E I_{z} v^{I V}+N\left(v+v_{o}\right)^{I I}+M_{y}\left(\theta+\theta_{o}\right)^{I I}=0 \\
& E I_{y} w^{I V}+N\left(w+w_{o}\right)^{I I}+M_{z}(\theta+\theta)^{I I}=0 \\
& E I_{w} \theta^{I V}-G I_{t} \theta^{I I}+i_{s}^{2} N\left(\theta+\theta_{o}\right)^{I I}+M_{y}\left(v+v_{o}\right)^{I I}+ \\
& +M_{y}\left(w+w_{o}\right)^{I I}=0
\end{aligned}
$$

where:

$E \quad$ is the Young's modulus of elasticity,

$G \quad$ is the shear modulus,

$I_{y}, I_{z} \quad$ is the second moment of area about the $y-y$ or $z-z$ axis, respectively,

I is the St. Venant's torsional constant,

$I_{w} \quad$ is the warping torsional constant,

$N \quad$ is the normal compressive force,

$M_{y}, M_{z}$ are the bending moments about the $y-y$ and $z-z$ axis, respectively, induced due to eccentrically acting axial force; $\mathrm{M}_{\mathrm{y}}=\mathrm{Ne}_{\mathrm{z}}, \mathrm{M}_{\mathrm{z}}=\mathrm{Ne}_{\mathrm{y}}$

\footnotetext{
* ${ }^{1}$ Josef Vican, ${ }^{1}$ Peter Janik, ${ }^{2}$ Ruzica Nikolic

${ }^{1}$ Department of Structures and Bridges, Faculty of Civil Engineering, University of Zilina, Slovakia

${ }^{2}$ University of Kragujevac, Faculty of Mechanical Engineering, Kragujevac, Serbia

E-mail: josef.vican@fstav.uniza.sk
} 
$i$ is the cross-sectional polar radius of gyration around to the centre of shear,

$v_{0}$ is the initial deflection of a member in the y-y axis direction,

$v$ is the deflection increment of a member in the $y-y$ axis direction,

$w$ is the deflection increment of a member in the $\mathrm{z}-\mathrm{z}$ axis direction,

$w_{o}$ is the initial deflection of a member in the $\mathrm{z}-\mathrm{z}$ axis direction,

$q_{o}$ is the angle of the initial cross-sectional rotation of a member about the $\mathrm{x}$-x axis,

$q$ is the angle increment of cross-sectional rotation of a member about the $\mathrm{x}-\mathrm{x}$ axis.

The analytical solution of the above described system of differential equations was presented, e. g., in [4] together with the comparison of the results experimentally obtained by Chuvikin [5] and results determined using numerical analyses by means of software ANSYS. A great attention was paid to the problem of the beam-column resistance verification in the frame of preparation of the European standard [1]. Conclusions and results of many numerical and experimental analyses and studies, worked up in the field of the above mentioned area, were summarized in [6] and [7] in comparison to the approach proposed in standard [1]. There is more information related to theoretical background of the beam-column resistance verification and solved examples according to rules for reliability verification of beam-column recommended in [1] using the method of substitute member based on the interaction formulas respecting the second-order theory.

\section{Experimental analysis}

\subsection{Test samples and testing description}

The formulas for verification of the beam-column resistances in standard [1] are based on the behavior of the single-span isolated members with simply supported end conditions. Therefore, the maximum bending moments in the two principal cross-section planes of loading are situated within the beam-column span. In the cases of other end conditions, the application of standard formulas for substitute members is rather complicated and extreme care should by taken when applying them to members integrated in frames. Accordingly, the experimental analyses supported by the numerical calculations were proposed to verify this general case approximated in both types of analyses, by the model of pinned-fixed beam-column. The main objective of the experimental investigation was to determine the actual ultimate resistances of tested beam-columns subjected to compressive axial force in combination with the applied end bending moments due to the axial force eccentricity and also to verify the suitability and correctness of standard approaches to the assessment of beam- column resistance in accordance with [1]. Consequently, four sets of beam-column samples, designated as A, B, C and D according to the type and eccentricity magnitude, were investigated in laboratory of the Department of structures and bridges. Each set of samples comprised three beam-columns made of IPE 120. Due to laboratory space and testing device arrangement, all samples had equal length of $1400 \mathrm{~mm}$ introducing beam-column relative slenderness of $\bar{\lambda}=0.82$ respecting the model of pinned-fixed beam-column. Beam-columns of set A were tested with the zero eccentricity of axial force (centrically loaded beam-columns), samples of set B had the preliminary measured eccentricity of the axial force of $e_{y}=32.2 \mathrm{~mm}$ in the $\mathrm{y}$-axis direction, samples of set $\mathrm{C}$ had the eccentricity of $e_{z}=52.2 \mathrm{~mm}$ measured in the z-axis direction and beam-columns of set $\mathrm{D}$ had the both type of eccentricities of axial force, i.e. $e_{v}=32.2 \mathrm{~mm}$ and $e_{z}=55.2 \mathrm{~mm}$ measured before experimental testing. The fixed edges were situated at the bottom ends of the vertically tested samples while the hinged boundary conditions were simulated at the tops of samples using the specially adjusted device. Both beamcolumns ends were equipped with the end-plates of $30 \mathrm{~mm}$ (at the bottom) or $20 \mathrm{~mm}$ (at the top) thick ensuring the zero warping deformations of beam-column edges.

Before testing, the actual geometrical and material characteristics of the beam-columns were determined and evaluated statistically. Concurrently, the initial bow imperfections in direction of both axes were measured by means of geodetic method and its measured amplitudes reached values of $L / 3860$ in the $\mathrm{z}$-axis direction and $L / 5250$ in the $\mathrm{y}$-axis direction. Using the obtained average material and geometric characteristics of tested beam-column (the yield strength $f_{y}=300 \mathrm{MPa}$, the cross-section area $A=1382.86 \mathrm{~mm}^{2}$, the second moments of cross-section area $I_{v}=3.308 .10^{6} \mathrm{~mm}^{4}, I_{z}=2.86 .10^{5} \mathrm{~mm}^{4}$ ) and taking into account the measured actual initial member eccentricities and bow imperfections, the resistance verifications of all the tested samples were performed according to [1], to prepare the testing program.

The strains and lateral deflections in chosen beam-columns locations (see Fig. 1) were monitored using gauges 6/120 LY11 (HBM) and potentiometer sensors of deformations TR50 recorded by means of Spider 8, also enabling recording of the actual value of compressive force monitored by dynamometer situated at the top end of the tested beam-column. As it can be seen in Fig. 1, the measurement devices were located at distance of $50 \mathrm{~mm}$ (gauges) or $40 \mathrm{~mm}$ (deformation sensors), respectively from the end-plates to avoid local effects of test load. Test loading was organized in two stages. In the first stage, the loading force increments were chosen gradually by $20 \mathrm{kN}$ up to limit value of $100 \mathrm{kN}$ ensuring the elastic state of maximum stressed beam-column cross-section. After the limit loading force had been reached, the unloading of the tested beam-column followed. In the second testing stage, the loading force increments were added gradually by $20 \mathrm{kN}$ up to the limit value of $200 \mathrm{kN}$ when the test has continued by means of deformation increments by constant 
GAUGES

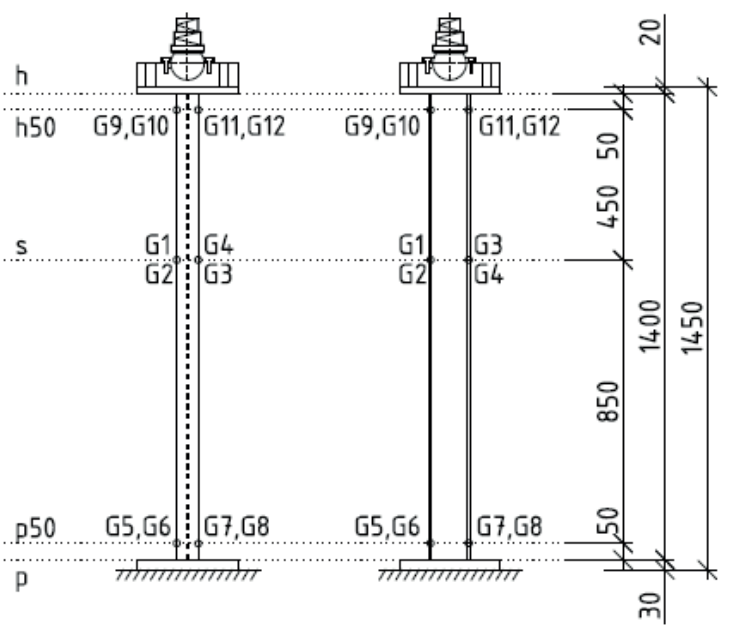

G OF CROSS-SECTION s<smiles>[13CH3]C([13CH3])[135I]</smiles>

G OF CROSS-SECTION p50

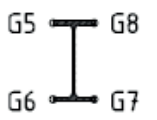

POTENTIOMETER SENSORS OF DEFORMATION

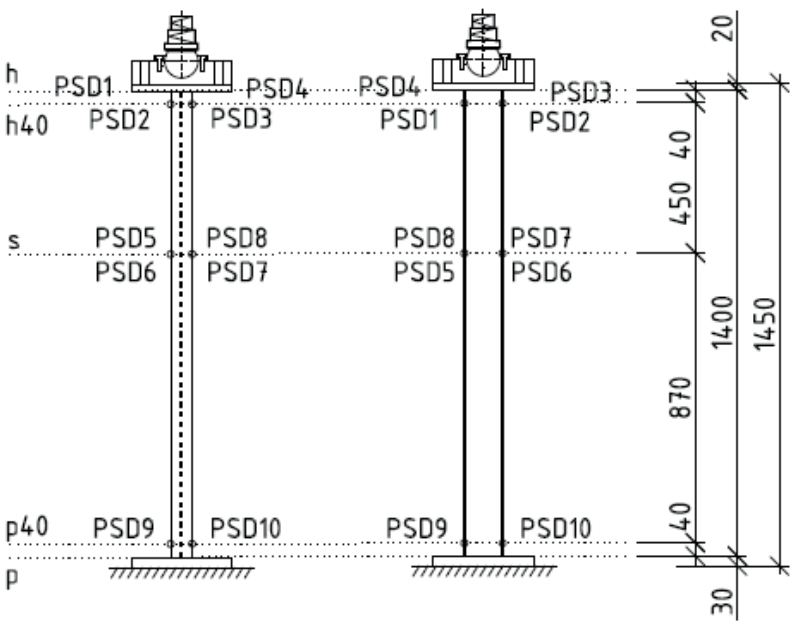

PSD OF CROSS-SECTION h40

PSD OF CROSS-SECTION S

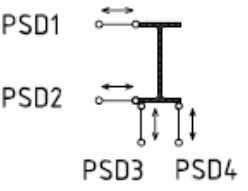

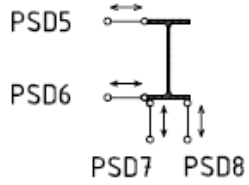

PSD OF CROSS-SECTION p 40

G OF CROSS-SECTION h50<smiles>CC(C)C=CC(C)C=[AsH2]</smiles>

Fig. 1 Laboratory samples of beam-columns with designated locations of measured strains and lateral deflections; G-gauges locations;

PSD - locations of deformation sensors

adding of $0.1 \mathrm{~mm}$, up to the beam-column collapse. The above described testing program was applied to all laboratory tested beam-columns. More information related to the voluminous experimental analysis can be found in [8].

\subsection{Results of experimental analysis}

The beam-column behavior was observed within the loading tests to identify the decisive steps of the member cross-section resistance. In the case of sample set $\mathrm{A}$, the first plasticization occurred in the cross-section p50 (see Fig. 1). Then the plasticization of cross-section $s$ followed and the plasticization of the cross-section $h 50$ was the last, very close to member collapse. In the case of sample set B, the first plasticization occurred in the cross-section $h 50$ (see Fig. 1).

Then the plasticization of the cross-section $p 50$ followed and the plasticization of the cross-section $s$ was the last. Results of tested samples of set $\mathrm{C}$ and $\mathrm{D}$ showed that the first plasticization occurred in the cross-section $h 50$, then the plasticization of the cross-section $s$ followed and the plasticization of the cross-section p50 was the last. Due to bending moment effects, the greater plastic reserves were indicated in the case of all tested beamcolumns compared to the centrically loaded samples of set A. Experimentally investigated ultimate resistances defined by the member collapses are presented in Table 1.

Ultimate resistances $N_{u}$ of tested members

Table 1

\begin{tabular}{|c|c|c|c|c|c|c|c|}
\hline Designation & $\mathbf{N}_{\mathrm{u}}[\mathbf{k N}]$ & Designation & $\mathbf{N}_{\mathrm{u}}[\mathbf{k N}]$ & Designation & $\mathbf{N}_{\mathrm{u}}[\mathbf{k N}]$ & Designation & $\mathbf{N}_{\mathrm{u}}[\mathbf{k N}]$ \\
\hline A1 & 346.0 & $\mathbf{B 1}$ & 133.0 & $\mathbf{C 1}$ & 221.0 & D1 & 107.0 \\
\hline A2 & 345.0 & $\mathbf{B 2}$ & 149.0 & $\mathbf{C 2}$ & 220.0 & D2 & 114.0 \\
\hline A3 & 341.0 & $\mathbf{B 3}$ & 148.0 & $\mathbf{C 3}$ & 211.0 & D3 & 112.0 \\
\hline Average & $\mathbf{3 4 4 . 0}$ & Average & $\mathbf{1 4 3 . 3}$ & Average & $\mathbf{2 1 7 . 3}$ & Average & $\mathbf{1 1 1 . 0}$ \\
\hline
\end{tabular}


Results of the experimental analyses were also used for evaluation of the actual force eccentricities and member bow imperfections. As it was mentioned above, the real imperfections of tested members were measured before testing to calculate more precisely the resistances of tested members. The evaluation of measured strains in the chosen locations gives more correct information related to the actual member imperfections including actual eccentricities of applied forces. From the view point of correct comparison of experimentally obtained resistances of tested samples to the numerically determined ones, the application of by these means evaluated imperfections into the numerical calculations is needed.

\section{Numerical analysis}

\subsection{Numerical modeling and results}

Generally, nine numerical models were created to simulate behavior of member samples A up to D. To plan the testing program, four numerical models were developed in the preparation stage before experimental testing. After experimental analysis, other five models were created taking into account real geometry and actual imperfections of tested members obtained by evaluating experimental tests. Due to large difference between the ultimate resistances of samples B1 and B2, B3 (see Table 1), the sample behavior of set B were modeled by means of two models respecting the mentioned differences.

Numerical models of tested members were developed in the working environment of the software Ansys -Workbench using actual geometric characteristics of tested members. The actual eccentricities, evaluated from the strain measurements were also implemented into the numerical models. Effects of residual stress were taken into account by means of equivalent geometric imperfection whose amplitude $\mathrm{e}_{0 \mathrm{e}}$ was calculated using standard expression for Perry factor in accordance with [1].

The bilinear material model with actual yield strength $f_{y}=300 \mathrm{MPa}$ obtained from material tests and the nominal value of the Young's elasticity modulus $E=210 \mathrm{GPa}$ was used to simulate material behavior. Effect of material strengthening was neglected because real beginning of material hardening reached almost $5 \%$ of measured strains while the strain corresponding to the actual material yield strength was $0.14 \%$. The numerical model was developed using the 3D finite elements Solid 186 and Solid 187, enabling geometrically and materially nonlinear analysis with imperfections included (GMNIA), [9]. Contacts were modeled using finite elements of Conta174, Targe 170 Surf154 and Combin14. An example of the applied numerical model created in working environment of the software Ansys -Workbench is shown in Fig. 2 for the case of tested sample set C.

Results of experimentally determined ultimate resistances of tested members A1, B1, C1 and D1 compared to numerically calculated ones using above described numerical models are presented in Table 2.

Ultimate resistances of tested members compared to numerically determined ones

Table 2

\begin{tabular}{|c|c|c|c|}
\hline Designation & $\begin{array}{c}\text { Experimental } \\
\text { analysis [kN] }\end{array}$ & $\begin{array}{c}\text { Numerical } \\
\text { analysis [kN] }\end{array}$ & Difference [\%] \\
\hline A1 & 346.0 & 346.16 & 0.05 \\
\hline B1 & 133.0 & 133.81 & 0.6 \\
\hline C1 & 221.0 & 226.88 & 2.6 \\
\hline D1 & 107.0 & 111.4 & 4.1 \\
\hline
\end{tabular}

It can be seen that the numerically obtained ultimate resistances of members are a little higher than experimentally achieved ones. That is probably caused due to applications of standard equivalent imperfections determined by means of standard expression for Perry factor in [1] which have the larger effects than real member's imperfections obtained from the measurements. Fig. 3 shows the deformation states of numerically analyzed members in the stage closely before member collapses (see Fig. 3).

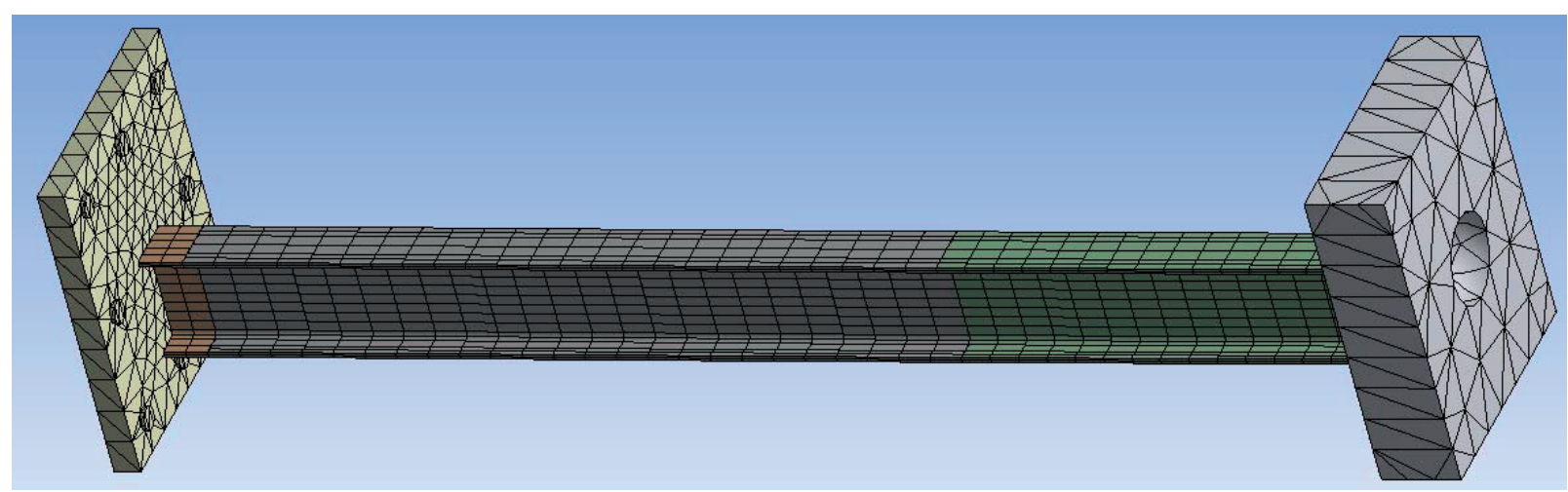

Fig 2 An example of the developed numerical model 


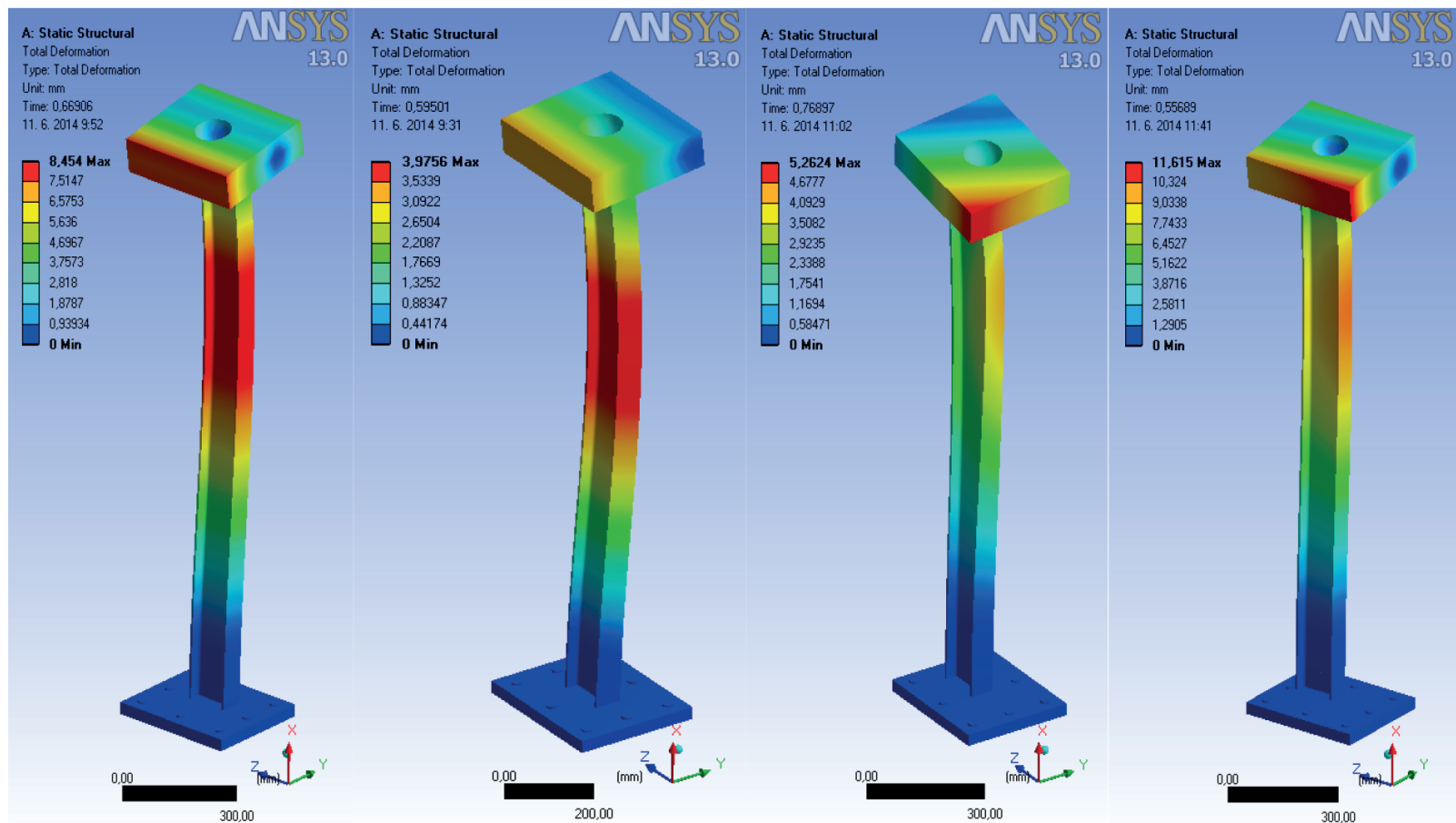

Fig. 3 Deformation states of numerically tested members in collapse stage (from A to D - from left to right)

\subsection{Comparison of horizontal deflections}

Values of recorded horizontal deflections of tested samples are compared to the numerically determined ones in Figs. 4 to 7 .

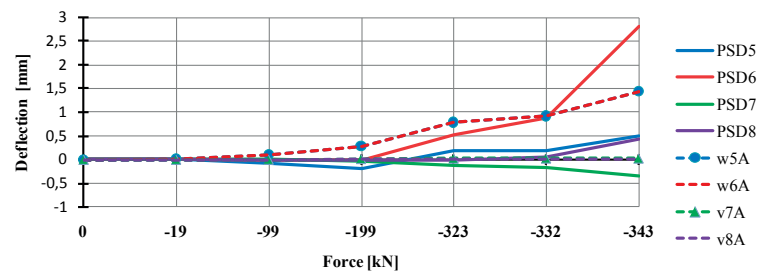

Fig. 4 Comparison of horizontal deflections in the cross-section $s$ of sample Al

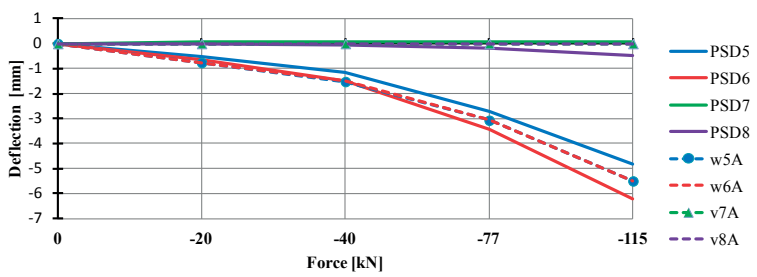

Fig. 5 Comparison of horizontal deflections in the cross-section $s$ of sample B1

Values of deflections calculated by means of above described numerical models are marked in Figs. 4 to 7 as w5A, w6A, v7A,
v8A and are corresponding with the values measured using deformation sensors designated as PSD5 up to PSD8 (see Figs. 4 to 7$)$.

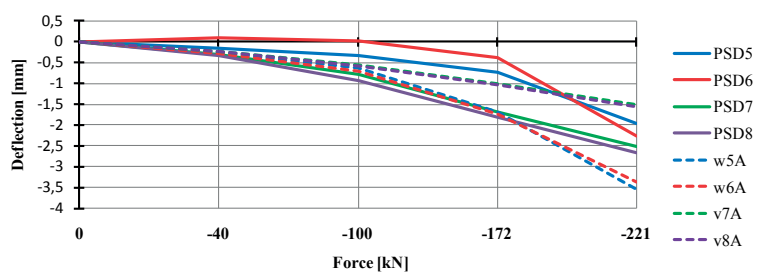

Fig. 6 Comparison of horizontal deflections in the cross-section $s$ of sample $\mathrm{Cl}$

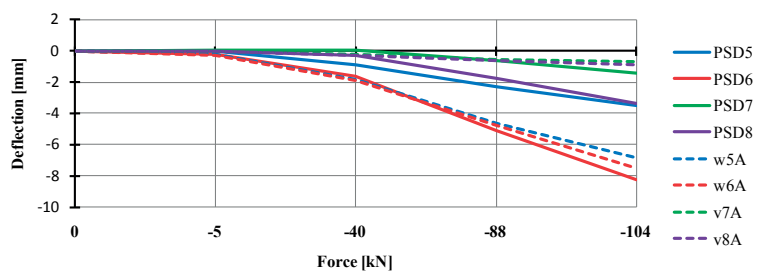

Fig. 7 Comparison of horizontal deflections in the cross-section $s$ of sample D1

From Figs. 4 to 7, the relative good accordance of measured deflections with the numerically determined ones is evident. 


\subsection{Comparison of strains}

Values of strains, measured within the experimental tests, were compared to the values of strains obtained using numerical calculations, respecting the elastic or elastic-plastic states of the member's cross - sections defined by means of the appropriate material model. Values of strains marked in Figs. 8 to 11 as $\varepsilon 1$ $\varepsilon 4$ are strains measured during experimental tests using gauges. Designations of $\varepsilon 1 \mathrm{~A}-\varepsilon 4 \mathrm{~A}$ correspond to values of strains obtained by means of the numerical calculations.

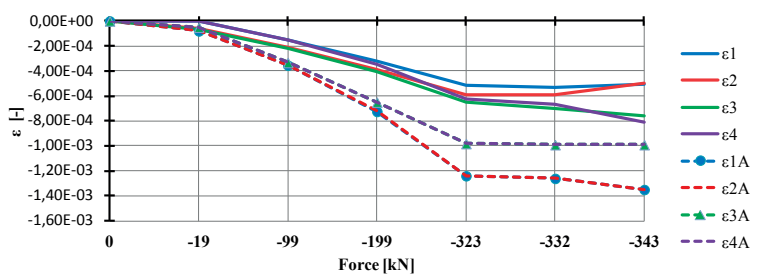

Fig. 8 Comparison of strains in cross-section s of member A1

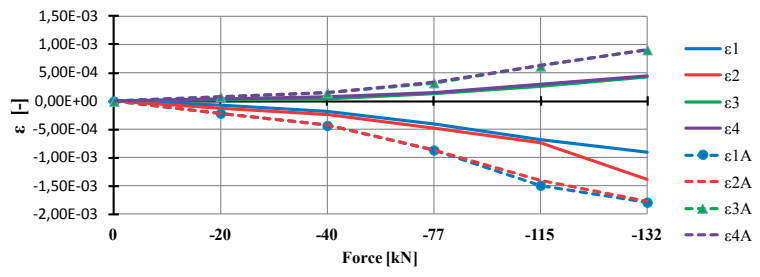

Fig. 9 Comparison of strains in cross-section s of member B1

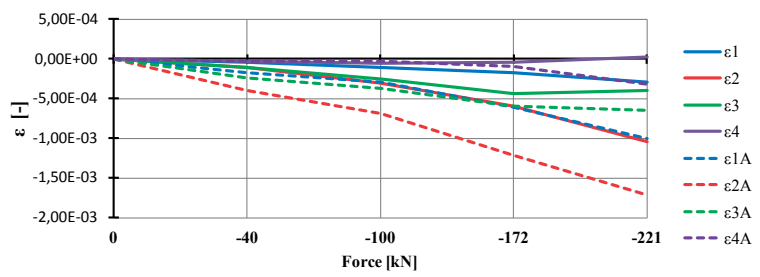

Fig. 10 Comparison of strains in cross-section s of member C1

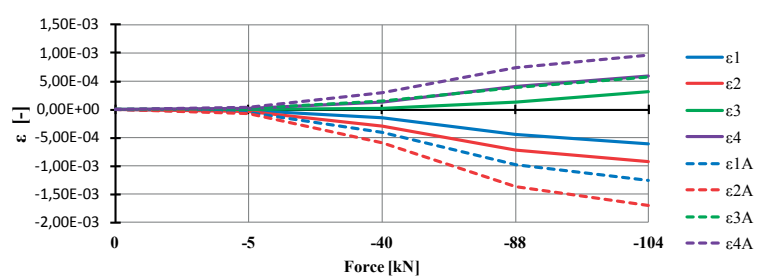

Fig. 11 Comparison of strains in cross-section s of member D1

Increase of strains showed similar trend in both analyses but measured values of strains are lower than numerically calculated ones (see Figs. 8 to 11). Difference of values could be caused by the greater effects of the second-order theory, considered in the numerical calculations compared to actual ones in the experimental analysis. This greater effect of the second order theory in numerical calculations proved that the standard equivalent geometric imperfection has a greater influence than the real member imperfections and eccentricities measured within testing.

\subsection{Comparison of experimental resistances to the standard ones}

The experimentally determined resistances of the tested samples were also compared to resistances defined using the standard approach according to [1]. In this standard two methods A and B for verification of the beam-column resistance are recommended. The resistances of the tested samples were determined from appropriate formulas valid for beam-column resistance verification under following assumptions:

- the buckling resistance only of the type A samples was assessed;

- the resistance of the B samples was determined without respecting the effects of lateral-torsional buckling;

- the assessment of the buckling resistances of the C and D samples was carried out with respecting the effects of the lateral-torsional buckling;

- $\quad$ the average value of the material yield strength $f_{y}=300 \mathrm{MPa}$ was considered,

- the nominal values of the Young's elasticity modulus $E=210$ $\mathrm{GPa}$ and shear modulus $G=81 \mathrm{GPa}$ were taken into account in calculations;

- the actual geometric characteristics of the tested samples obtained from the measurements were considered to calculate the standard resistances;

- the buckling lengths of the tested samples $C$ and D were taken as $L_{y}=L_{z}=980 \mathrm{~mm}$;

- the imperfection factors $\alpha_{I}=0.21$ for buckling in the z-axis direction and $\alpha_{1}=0.34$ for buckling in the y-axis direction were considered for calculation of initial bow amplitudes in the both member cross-section directions.

The obtained results of the resistances calculated according to methods A and B from the standard [1] are presented in Table 3 together with the resistances determined experimentally. The following designations are used in Table 3:

$e_{y} \quad$ is the eccentricity of the axial force in the y-axis direction;

$e_{z} \quad$ is the eccentricity of the axial force in the z-axis direction;

$N_{\text {exp }}$ is the experimentally determined ultimate resistance of tested members:

$N_{A}$ is the resistance of the tested members calculated by means of method $\mathrm{A}$ in accordance with [1];

$N_{B}$ is the resistance of the tested members calculated by means of method B in accordance with [1]. 
Comparison of experimentally determined resistances to the standard ones

Table 3

\begin{tabular}{|c|c|c|c|c|c|c|c|}
\hline \multirow{2}{*}{ Designation } & $\mathrm{e}_{\mathrm{z}}$ & $\mathrm{e}_{\mathrm{y}}$ & $\mathrm{N}_{\mathrm{exp} .}$ & $\mathrm{N}_{\mathrm{A}}$ & $\mathrm{N}_{\mathrm{B}}$ & $\mathrm{N}_{\mathrm{A}} / \mathrm{N}$ & $\mathrm{N}_{\mathrm{B}} / \mathrm{N}$ \\
\cline { 2 - 9 } & {$[\mathrm{mm}]$} & {$[\mathrm{mm}]$} & {$[\mathrm{kN}]$} & {$[\mathrm{kN}]$} & {$[\mathrm{kN}]$} & {$[\%]$} & {$[\%]$} \\
\hline $\mathrm{A} 1$ & 0.00 & 0.00 & 323.00 & 295.5 & 295.5 & 91.5 & 91.5 \\
\hline $\mathrm{B} 1$ & 0.00 & 13.00 & 133.00 & 126.00 & 131.00 & 98.5 & 89.5 \\
\hline $\mathrm{C} 1$ & 22.65 & 2.36 & 221.00 & 214.00 & 196.00 & 96.8 & 88.7 \\
\hline $\mathrm{D} 1$ & 36.50 & 18.34 & 106.60 & 122.00 & 110.00 & 114.4 & 103.2 \\
\hline
\end{tabular}

Besides the sample of type D1, the comparison of the member resistances presented in Table 3 proves very good compliance of experimentally determined resistances with those calculated according to approaches recommended in [1] (see Table 3). The member resistances calculated by means of method A are closer to the experimental results than resistances determined according to method B. This conclusion corresponds to the theoretical background of both methods whereas the method A is generally considered as the more precise approach to the beam-column resistance verification.

\section{Conclusions}

The paper presents the results of voluminous experimental analysis of resistances of the pinned-fixed beam-columns subjected to centrically (samples of set A) and eccentrically (samples of sets $\mathrm{B}$ to $\mathrm{D}$ ) acting axial force and their comparison to the numerically determined ones using GMNIA. Results of comparison proved very good correspondence of numerical calculations with the experimental tests; thus the developed numerical models could be used for further parametric studies to obtain more information about actual behavior of those complicated structural members. The standard approach designated as method A [1] also proved relatively good compliance due to its more precise but also more complicated formulation. Moreover, the experimental analysis confirmed the correctness of theory related to importance of the point with the maximum effect of the second-order theory [8].

\section{Acknowledgements}

This work was supported by the Slovak Research and Development Agency under contract APVV-0106-11 and by Research Project No. 1/0364/12 of Slovak Grant Agency. Moreover, the research presented here was supported by the European Regional Development Fund and the Slovak state budget for the project "Research Centre of University of Zilina", ITMS 26220220183.

\section{References}

[1] STN EN 1993-1-1. Eurocode 3: Design of Steel Structures - Part 1-1: General Rules and Rules for Buildings, CEN Brussels, May 2005.

[2] VLASOV, V.Z.: Thin-walled Elastic Members, SNTL, Prague, 1962.

[3] BREZINA, V.: Buckling Resistance of Metal Members and Beams, Czechoslovak academy of sciences : Prague, 1962.

[4] VICAN, J., JANIK, P.: Beam-column Resistance According to Slovak Standard and Eurocodes, Communications - Scientific Letters of the University of Zilina, 15 (2013), pp. 21-27.

[5] GALAMBOS, T. V.: Review of Tests on Biaxially Loaded Steel Wide-Flange Beam-Columns. Lehigh University, April, 1963.

[6] BOISSONNADE, N. et al.: Rules for Member Stability in EN 1993-1-1, Background Documentation and Design Guidelines, ECCS 2006.

[7] SIMOES DA SILVA, L., SIMOES, R., GERVASIO, H.: Design of Steel Structures - Eurocode 3: Design of Steel Structures Part 1-1: General Rules and Rules for Buildings. Mem Martins : Multicomp Lda, 2010.

[8] JANIK, P.: Resistance of Beam-column Subjected to Transversal Load and Axial Force, PhD. Thesis, University of Zilina, 2014.

[9] KALA, Z., KALA, J.: Respecting the Influence of Geometrical and Material Imperfections of Steel Beam when Calculating their Loadcarrying Capacity, Stability and Ductility of Steel Structures, Budapest : Akademiai Kiado, 2002, pp. 103-110. 